\title{
Lymphocytic reaction in kidneys following snake bites: a histological descriptive report
}

\author{
Neelakanthi V I Ratnatunga ${ }^{1}$, H M Nalaka J Herath ${ }^{2}$, Abdul W M Wazil ${ }^{2}$, D Tilak D J Abeysekera ${ }^{2}$
}

Journal of the Ceylon College of Physicians, 2013, 44, 28-31

\begin{abstract}
Acute kidney lesions following snake bites are well documented. We reported the development of chronic kidney disease following snake bites in a Sri Lankan cohort ${ }^{2}$. This retrospective study documents the histopathology of kidneys following snake bites, on a time line basis, with special reference to the presence of $\mathrm{T}$ and $\mathrm{B}$ lymphocytes. $\mathrm{T}$ lymphocytes, predominantly $\mathrm{CD} 3$, were present in the renal interstitium from the $1^{\text {st }}$ week after the bite to as late as 90 weeks, long after cessation of tubular and glomerular necrosis. This finding suggests the possibility of continuing renal damage by the process of interstitial inflammation and fibrosis mediated by these cells.
\end{abstract}

\section{Introduction}

Kidney lesions reported following venomous snake bites are acute tubular necrosis, cortical necrosis, glomerulitis, mesangiolysis, proliferative glomerular nephritis and interstitial nephritis ${ }^{1}$. Studies done in Sri Lanka, report similar pathology ${ }^{2,3}$. Although acute interstitial nephritis following venomous snake bites has been reported, the occurrence of chronic interstitial inflammation was not well established ${ }^{1,4,5}$. We recently reported the development of chronic kidney disease in $37 \%$ of people who had severe acute kidney injury, following snake bites $^{2}$. Thus, the possibility of continuing kidney injury after snake bites is a strong reality. Our objective was to document, on a time line basis, the pathological lesions in the kidneys, with special reference to infiltration of $B$ and $T$ lymphocytes in snake bite victims in Sri Lanka.

\section{Method}

This is a retrospective analysis of the renal histopathology, obtained from archival slides and blocks of renal biopsies and postmortem specimens of victims of Russell (n-8), Hump Nosed Viper (n-5), and unknown snake bite $(n-10)$ received in the

\footnotetext{
${ }^{1}$ Department of Pathology, Faculty of Medicine, University of Peradeniya, Sri Lanka.

${ }^{2}$ Nephrology and Transplant Unit, Teaching Hospital, Kandy, Sri Lanka.
}

Department of Pathology, University of Peradeniya during the period of January 2000 to 2012. Ethical approval was obtained from the ethical committee of the University of Peradeniya. There were renal tissues of 23 victims of snake bites from 3 categories;

1. Autopsies on those succumbing to acute kidney injury (n-4),

2. Biopsies done to assess the prognosis of those who were dialysis dependent (n-10),

3. Biopsies done for evaluation of unexplained renal failure with active urinary sediments and past history of snake bite (n-9).

Haematoxylin and eosin stained sections and 3 stage streptavidin biotin immunoperoxidase stained sections for identifying CD3, and CD8 positive $T$ lymphocytes, and CD20 positive B lymphocytes were studied. Glomerular, tubular, interstitial and vascular lesions were documented by light microscopy (table 1). A semi quantitative assessment of these lesions was done. Immunofluoresence was done on a limited number of cases.

\section{Results}

Glomerular and tubular necrosis was seen alone or in combination in acute stages. Later, tubulointerstitial disease was more prominent. Intense infiltration of lymphocytes was the key histological feature from one week to ninety weeks. Arteriolar sclerosis was a late feature which starts from 40th week. Hypertension (n-9) and diabetes (n-2) was noted in eleven patients. Renal histopathology compatible with diabetic nephropathy was not observed in this cohort.

Glomerular lesions - Glomerular necrosis was seen up to the 6 th week after the bite and glomerular sclerosis was seen from then onwards (table 1).

Tubular lesions - Tubulitis was seen from the 5th week onwards. Acute tubular necrosis was seen up to the 12th week after the bite, and did not persist thereafter. Haemosiderin in the tubular epithelial cells was seen up to 20 weeks after the bite, and indicated the occurrence of haemoglobinura probably due to intravascular haemolysis, during this period (table 1). 


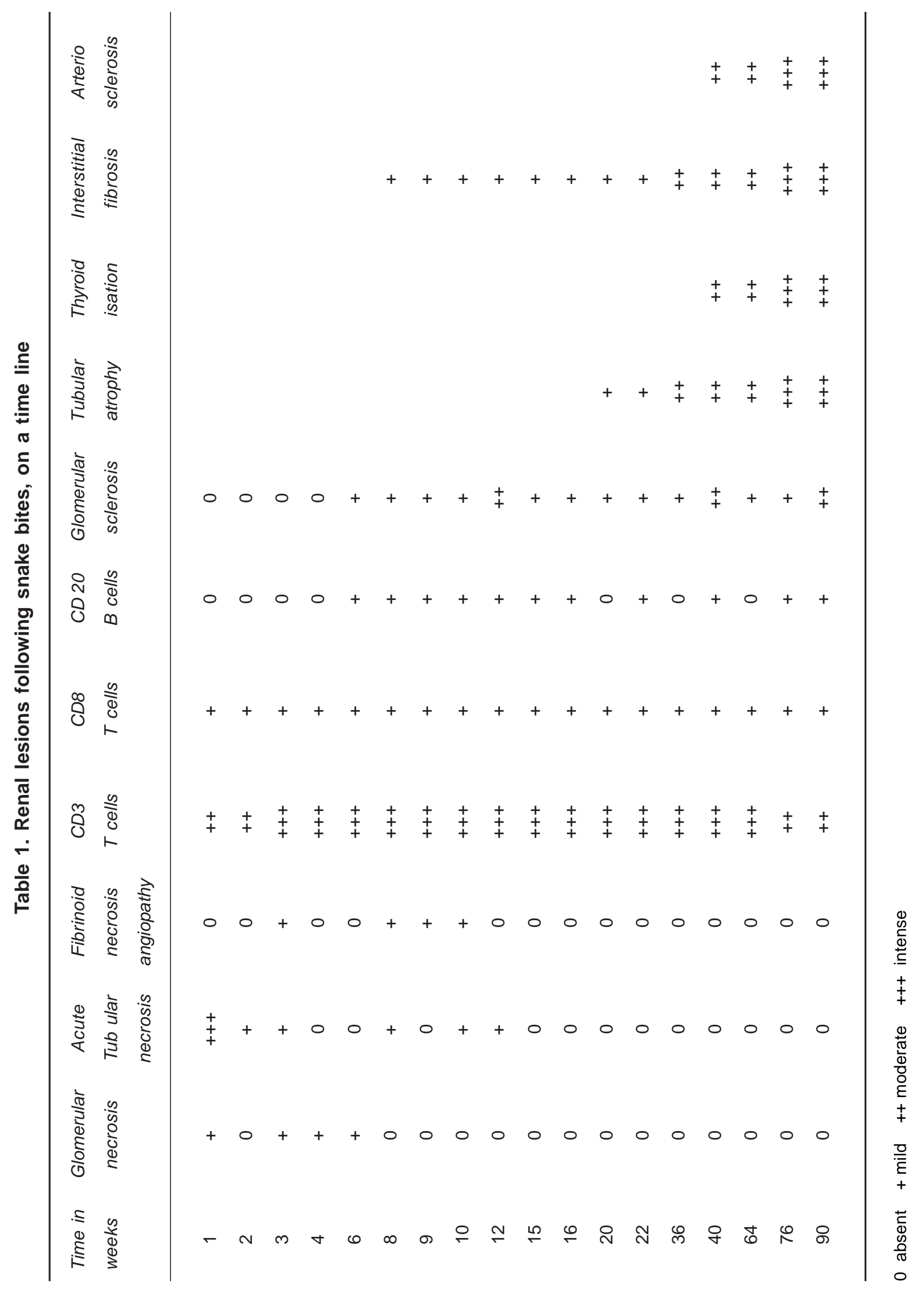


Figure 1. Eight weeks after bite

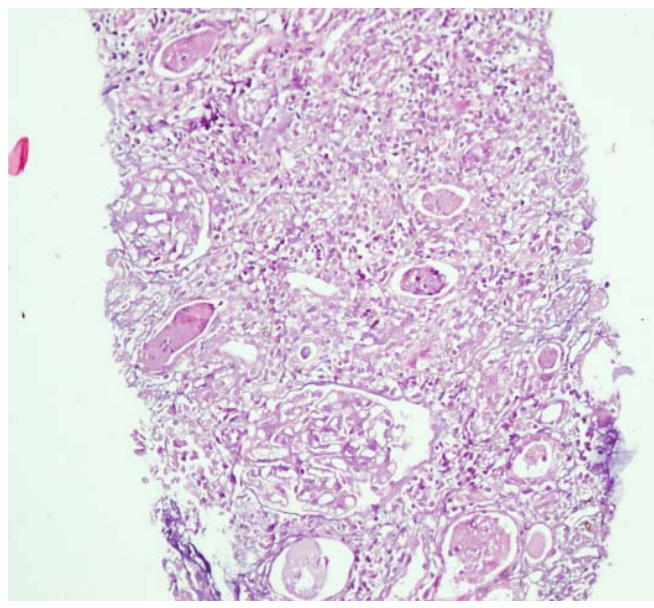

Figure 1.1 Haematoxylin and Eosion $X 20$ shows intense interstitial infiltration with lymphocytes.

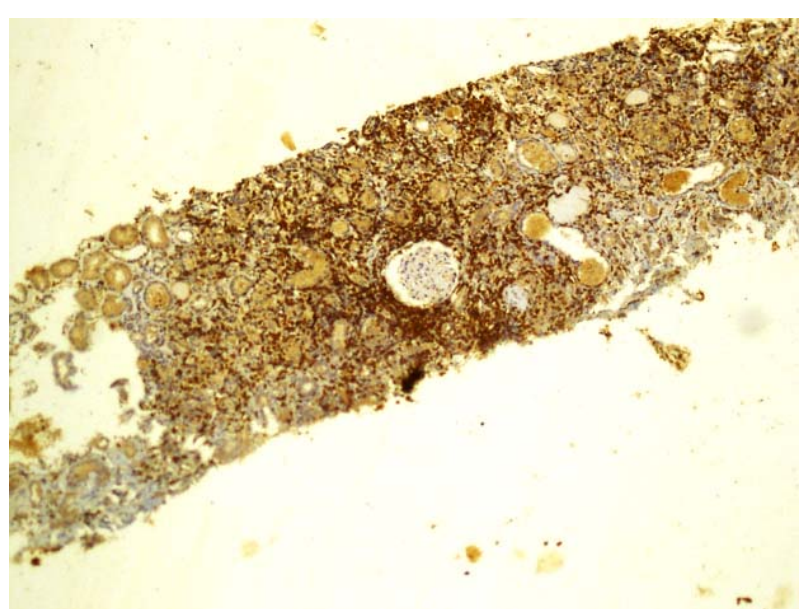

Figure 1.2 The majority of the cells are CD3 T lymphocytes.

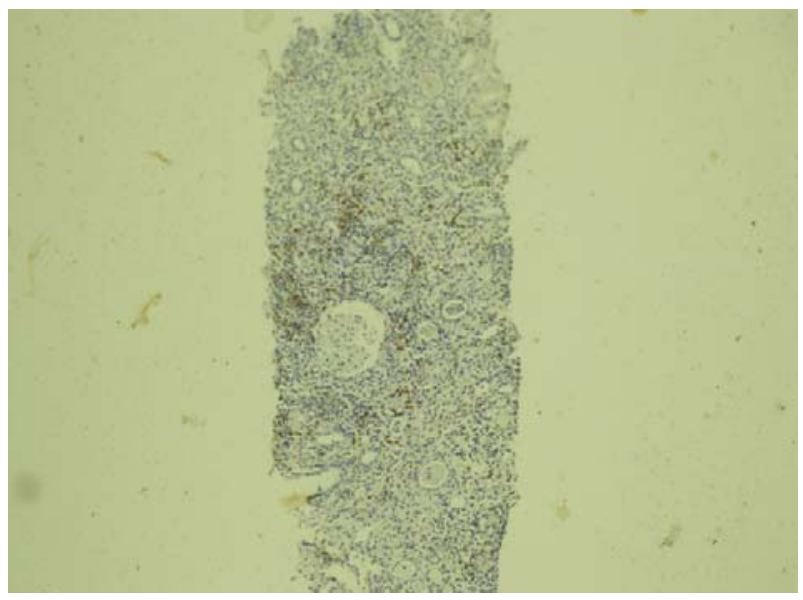

Figure 1.3 Sparse small collections of CD20B lymphocytes are seen.
Figure 2. Twenty two weeks after bite

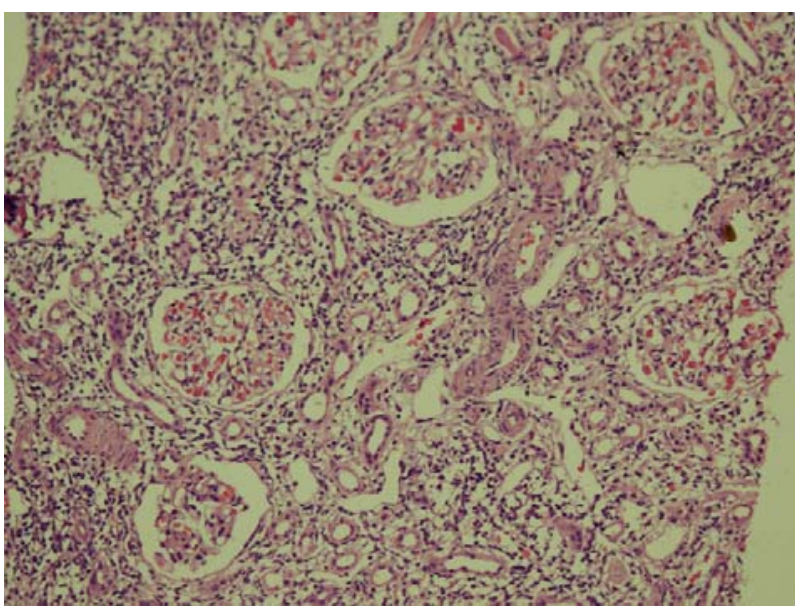

Figure 2.1 Haematoxylin and eosion $X 20$ shows persisting intense interstitial infiltrate with lymphocytes.

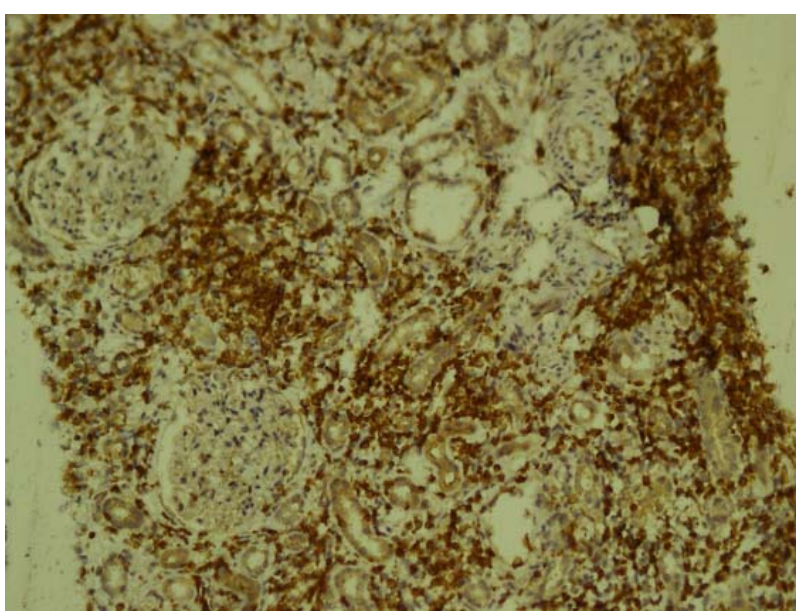

Figure 2.2 The majority of the cells are CD3 T lymphocytes.

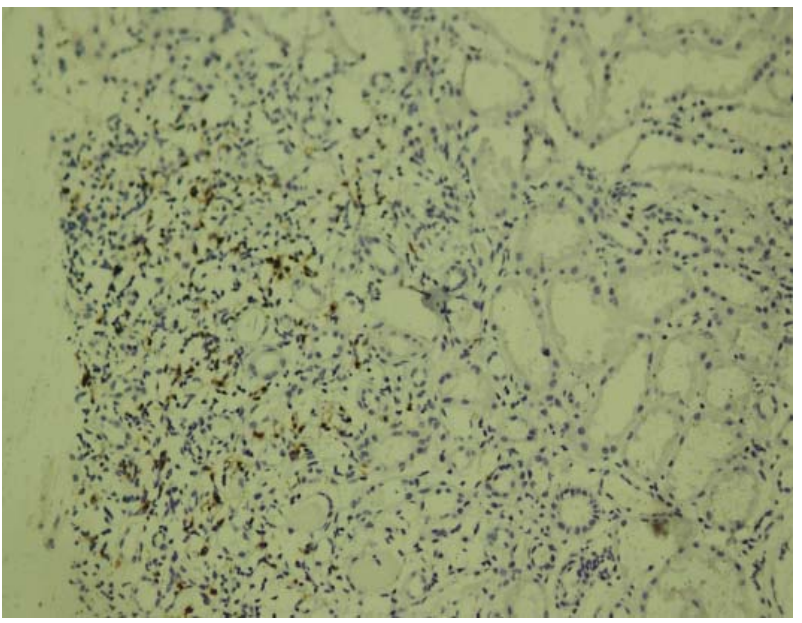

Figure 2.3 The CD 20 B cells are sparse.

Journal of the Ceylon College of Physicians 
Interstitial lesions - The intense interstitial infiltration of CD3 lymphocytes is striking from the first week after the bite which persists as late as the $90^{\text {th }}$ week. CD 8 lymphocytes were seen in lesser numbers. B lymphocytes appeared from the $6^{\text {th }}$ week after the bite, and were variably present thereafter but not as intensely as the CD3 lymphocytes. In addition to the lymphocytes, neutrophils, eosinophils and plasma cells were seen up to 20 weeks. Varying proportions of interstitial fibrosis was seen from the $7^{\text {th }}$ week and tubular atrophy from 20 weeks onwards. Dilatation of tubules with protein casts giving the classic thyroidisation of the renal parenchyma which indicates extensive renal scarring was seen 40 weeks after the bite (table 1 ).

Vascular lesions - Vascular fibrinoid necrosis was seen from the 3rd to 10th week. Arteriosclerosis was seen after the 40th week (table 1).

\section{Discussion}

Most of our histological findings follow the pattern described in literature ${ }^{1}$. However, we found that persistence of intense interstitial infiltration of CD3 lymphocytes up to 90 weeks was striking in this study. This was out of proportion to the number of sclerosed glomeruli. CD 8 lymphocytes were seen in lesser numbers. B lymphocytes appeared from the 6 th week after the bite, and were variably present thereafter but not as intensely as the CD3 lymphocytes.

Development of CKD after AKI is well described ${ }^{2}$ in literature (depending on the severity of $A K I)$. This study suggests the possibility of continuing renal damage by the process of interstitial inflammation and fibrosis mediated by these activated CD3 lymphocytes.

There are some limitations in this study. Identification of the culprit snake (RV or HNV) had not been recorded in all cases. Because of that reason we tried to document the renal histopathology in general to snake bite in time line basis. Secondly, some of these patients did not have pre-morbid renal functions. There is a small possibility that they have got CKD before this acute insult. Though renal histopathology is straightforward in acute stage of snake bite, coexisting CKDu may be difficult to differentiate in chronic cases.

\section{Conclusion}

Acute tubular necrosis, glomerular necrosis, and vascular fibrinoid necrosis are acute lesions, and they persist up to 12th week after the snake bite. The infiltration of CD3 lymphocytes into the kidneys early in the course after the snake bite, and persisting thereafter even up to 90 weeks, is a striking feature, and could suggest a possible cause for the occurrence of CKD after snake bite.

\section{Acknowledgements}

We thank Mr.S. Chandraprabha and Mrs.Nadeeka Herath for their technical assistance and Dr Kosala Weerakoon for his valuable comments.

\section{References}

1. Sitprija V. Snakebite nephropathy. Nephrology 2006; 11: 442-8.

2. Herath HMNJ, Wazil AWM, Abeysekera DTDJ, et al.Chronic kidney disease in snake envenomed patients with acute kidney injury in Sri Lanka: a descriptive study. Postgrad ed J 2012 doi10.1136/postgradmedj-2011-130225.

3. Kularatne SA, Ratnatunga N. Severe systemic effects of Merrem's humb-nosed viper bite. Ceylon Med J 1999; 44: 169-70.

4. Indraprasit S, Boonpucknavig V. Acute interstitial nephritis after a Russell's viper bite. Clin. Nephrol.1986; 15: 111.

5. Sitprija V, Suvanpha R, Pochanugool C, et al. Acute interstitial nephritis in snake bite. Am J Trop Med Hyg 1982 ; 31: 408-10. 